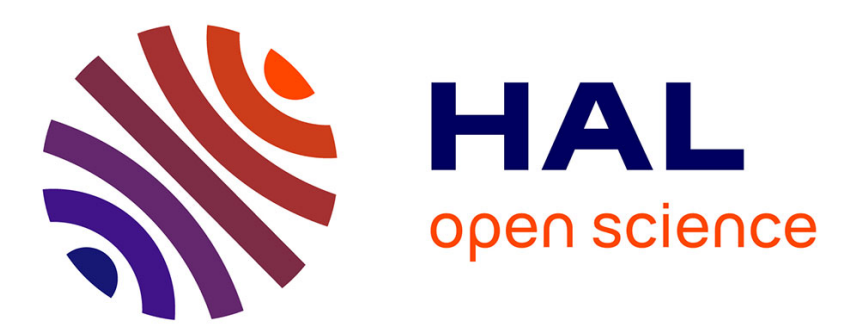

\title{
The Escherichia coli thioredoxin homolog YbbN/Trxsc is a chaperone and a weak protein oxidoreductase.
}

\author{
Thérèse Caldas, Abderrahim Malki, Renée Kern, Jad Abdallah, Gilbert
}

Richarme

\section{- To cite this version: \\ Thérèse Caldas, Abderrahim Malki, Renée Kern, Jad Abdallah, Gilbert Richarme. The Escherichia coli thioredoxin homolog YbbN/Trxsc is a chaperone and a weak protein oxidoreductase.. Biochemical and Biophysical Research Communications, 2006, 343 (3), pp.780. 10.1016/j.bbrc.2006.03.028 . hal- 00021859}

\section{HAL Id: hal-00021859 \\ https://hal.science/hal-00021859}

Submitted on 27 Mar 2006

HAL is a multi-disciplinary open access archive for the deposit and dissemination of scientific research documents, whether they are published or not. The documents may come from teaching and research institutions in France or abroad, or from public or private research centers.
L'archive ouverte pluridisciplinaire HAL, est destinée au dépôt et à la diffusion de documents scientifiques de niveau recherche, publiés ou non, émanant des établissements d'enseignement et de recherche français ou étrangers, des laboratoires publics ou privés. 
THE E. COLI THIOREDOXIN HOMOLOG YbbN / TRXSC IS A CHAPERONE AND A WEAK PROTEIN OXIDOREDUCTASE

Thérèse Caldas, Abderrahim Malki, Renée Kern, Jad Abdallah and Gilbert Richarme*

Stress molecules, Institut Jacques Monod, Université Paris 7, 2 place Jussieu, 75005 Paris, France

*Corresponding author

Tel 330144275098

Fax 330144275716

Email: richarme@,ccr.jussieu.fr

*Corresponding author

Running title : YbbN chaperone and oxidoreductase.

Keywords : YbbN / chaperone / oxidoreductase / SXXC / heat shock.

Abbreviations: Hsp: heat shock protein ; R-CMLA: reduced carboxymethyl ${ }_{\alpha}$-lactalbumin ; PDI: protein disulfide isomerase, DTNB: 5,5'-dithiobis(2-nitrobenzoic acid), DTT: dithiothreitol. 
ABSTRACT

Escherichia coli contains two thioredoxins, Trx1 and Trx2, and a thioredoxin-like protein, YbbN which presents a strong homology in its N-terminal part with thioredoxin1 and 2. YbbN, however, does not possess the canonical Cys-x-x-Cys active site of thioredoxins, but instead a Ser-x-x-Cys site. In addition to Cys-38, located in the SxxC site, it contains a second cysteine, Cys-63, close to Cys-38 in the 3D model. Cys-38 and Cys-63 undergo an oxidoreduction process, suggesting that $\mathrm{YbbN}$ functions with two redox cysteines. Accordingly, YbbN catalyzes the oxidation of reduced RNase and the isomerization of scrambled RNase. Moreover, upon oxidation, its oligomeric state changes from dimers to tetramers and higher oligomers. YbbN also possesses chaperone properties, promoting protein folding after urea denaturation and forming complexes with unfolded proteins. This is the first biochemical characterization of a member of the YbbN class of bacterial thioredoxin-like proteins, and in vivo experiments will allow to determine the importance of its redox and chaperone properties in the cellular physiology. 


\section{INTRODUCTION}

E. coli contains two thioredoxins, $\operatorname{Trx} 1$ (the $\operatorname{tr} x A$ gene product) and $\operatorname{Trx} 2$ (the $\operatorname{tr} x C$ gene product) [1] which are equivalent for most of the in vivo functions tested [2-3], and a thioredoxin-like protein, YbbN, which presents a strong homology in its $12 \mathrm{Kda} \mathrm{N}$-terminal part with thioredoxin 1 and 2, but possesses a $20 \mathrm{kDa}$ C-terminal part of unknown function. Thioredoxin 1 is a small soluble protein of $12 \mathrm{kDa}$ with a Cys-X-XCys active site, capable of catalyzing thiol-disulfide redox reactions. The thioredoxin system is ubiquitous from bacteria to man [1]. In this system, thioredoxin reductase uses the reducing potential of NADPH to maintain thioredoxin in a reduced state, so that thioredoxin can in turn reduce substrate proteins. The thioredoxin system is a hydrogen donor for several reductive enzymes such as ribonucleotide reductase, methionine sulfoxide reductase and sulfate reductase [4]. It also plays a key role in maintaining cytoplasmic proteins in a reduced state [5-6] (unlike cytoplasmic proteins which are reduced, extracytoplasmic proteins are oxidized by PDI in eukaryotes and Dsb enzymes in bacteria [7]). The other major factor generally responsible for the low redox potential inside cells is glutathione, which is maintained in its reduced state by NADPH and glutathione reductase [4]. Glutathione-dependent disulfide reductions are catalyzed by glutaredoxins whose functions partially overlap those of thioredoxin $[4,8]$.

Thioredoxin 2 is a 139 aminoacids thioredoxin of $15.5 \mathrm{kDa}$ which contains two distinct domains: The first, an N-terminal domain of 32 aminoacids contains two CxxC motifs which are devoid of any redox function, bind zinc with high affinity and are probably involved in the regulation of its activity. The second, a Cterminal domain, with the conserved WCGPC site, presents a strong homology to the prokaryotic thioredoxins [9]. Thioredoxin 2 is involved in the oxidative stress response, and its expression is induced in response to elevated hydrogen peroxide levels [3].

The $31 \mathrm{kDa}$ thioredoxin homolog YbbN presents a strong homology, in its N-terminal part (111 aminoacids), with thioredoxin 1 and thioredoxin 2 (see below), but possesses a C-terminal part (173 aminoacids) of unknown function. Despite its extensive homology with thioredoxins, YbbN does not possess the canonical Cys-X-X-Cys active site that catalyzes protein disulfide interchange, but instead a Ser-X-X-Cys site. Consequently, we propose to rename it Trxsc (for thioredoxin with a Ser-X-X-Cys site). Although CxxC is the major redox motif used for formation, reduction, and isomerization of disulfide bonds, CxxC-derived motifs, such as CxxS, SxxC, CxxT, TxxC are found in several redox proteins, including glutaredoxins, arsenate reductases, methionine sulfoxide reductases $(\mathrm{CxxS})$, peroxiredoxins (SxxC, TxxC), glutathione peroxidases (CxxT) [10], and in several uncharacterized thioredoxin-like proteins.

In this study, we show that, in addition to the Cys-38 at the SxxC site, Trxsc contains a second cysteine, Cys63 which is close to Cys-38 in the 3D model. Both Cys-38 and Cys-63 are titratable with DTNB in the native, reduced protein, and form a disulfide upon oxidation. In consistence with this result, YbbN displays protein disulfide oxidoreductase activities. YbbN also possesses chaperone propeties. This is the first biochemical characterization of a member of the YbbN class of bacterial thioredoxin-like proteins. 
MATERIALS AND METHODS

Bacterial Strains, Plasmids, YbbN/Trxsc expression and purification. We placed the YbbN/Trxsc open reading frame under transcriptional control of the bacteriophage $\mathrm{T} 7$ promoter and lac operator. The E. coli B/r strain AD494 (ara leu7967 lacX74 phoA phoR-malF3 F' [lac ${ }^{+}$(lacIq) pro] trxB::kan [DE3] pLysS was purchased from Novagen. The gene coding for YbbN/Trxsc was inserted in plasmid pET-21a (from Novagen). It was generated by amplifying the $y b b N$ gene from E.coli DNA by PCR using primers (5'GGTGGTTGCTCTTCACATATGTCAAAGAAAATATCGAC-3') (5' GGTGGTCTGGGATCCTCATCAATACAACAATGCATACAG-3'). The resulting product was digested with $N d e 1$ and BamH1, ligated to the pET-21a Nde1 and BamH1 backbone fragment, and transformed into strain BL21(DE3). BL21(DE3) transformants induced with IPTG accumulated large amounts of a soluble 35 $\mathrm{kDa}$ protein that matched the expected YbbN/Trxsc molecular weight of 32,000. Trxsc was first purified on a DEAE-Sephacel (Pharmacia) column equilibrated in $30 \mathrm{mM}$ Tris $\mathrm{pH}$ 8, $20 \mathrm{mM} \mathrm{NaCl}, 0.5 \mathrm{mM}$ EDTA, $1 \mathrm{mM}$ DTT at $20^{\circ} \mathrm{C}$, and eluted with a linear gradient of $20-400 \mathrm{mM} \mathrm{NaCl}$ in the same buffer. Trxsc was further purified on a hydroxyapatite column (Bio-Gel HTP from BioRad) equilibrated in $30 \mathrm{mM}$ Tris $\mathrm{pH} \mathrm{8,} 20 \mathrm{mM}$ $\mathrm{NaCl}, 0.5 \mathrm{mM}$ EDTA, $1 \mathrm{mM}$ DTT at $20^{\circ} \mathrm{C}$ and eluted with a linear gradient of $0-50 \mathrm{mM}$ sodium phosphate $\mathrm{pH} 8$ in the same buffer. YbbN/Trxsc was dialyzed for 2 hours against $30 \mathrm{mM}$ Tris $\mathrm{pH} 8,20 \mathrm{mM} \mathrm{NaCl}, 0.5$ $\mathrm{mM}$ EDTA, $1 \mathrm{mM}$ DTT at $20^{\circ} \mathrm{C}$, and stored at $-20^{\circ} \mathrm{C}$ in the same buffer.

Oligomeric forms of reduced and oxidized Trxsc. The molecular weight of native YbbN/Trxsc was determined by filtration of the protein on a TSK-G3000-SW HPLC gel permeation column (Hewlett Packard). The column was equilibrated in $20 \mathrm{mM}$ Tris $\mathrm{pH} 7.5,100 \mathrm{mM} \mathrm{NaCl}, 1 \mathrm{mM}$ DTT at $20^{\circ} \mathrm{C}$, loaded with $20 \mu 1$ of reduced or oxidized Trxsc $(2 \mathrm{mg} / \mathrm{ml})$ and eluted at a flow rate of $0.5 \mathrm{ml} / \mathrm{min}$. YbbN/Trxsc was detected by its absorbance at $280 \mathrm{~nm}$. Blue dextran, $\beta$-amylase, alcohol dehydrogenase, bovine serum albumin, carbonic anhydrase and vitamin B12 were used as molecular weight standards.

Preparation of reduced and oxidized Trxsc. $40 \mu \mathrm{l}$ of Trxsc $(15 \mathrm{mg} / \mathrm{ml})$ was incubated for $30 \mathrm{~min}$ with 10 $\mathrm{mM}$ dithiothreitol and isolated by gel filtration through a bio-Gel P10 column ( $2 \mathrm{ml}$ bed volume, Bio-Rad) equilibrated with oxygen-free $0.1 \mathrm{M}$ potassium phosphate $\mathrm{pH}$ 6.8, 1 mM EDTA [11-12]. SH was determined immediately by adding $10 \mu \mathrm{l}$ of each fraction to $100 \mu \mathrm{l}$ of $1 \mathrm{mM}$ DTNB, $50 \mathrm{mM}$ Tris $\mathrm{pH}$ 7.5. The number of SH groups per mol of Trxsc was calculated from the Trxsc concentration and from the absorbance at $412 \mathrm{~nm}$. Trxsc concentration was calculated from its absorbance at $280 \mathrm{~nm}$ with $\mathrm{A}_{1 \mathrm{mg} / \mathrm{ml}}=0.680$ calculated from tryptophan and tyrosine content. Oxidized Trxsc was obtained by incubating reduced Trxsc for 1 hour at $37^{\circ} \mathrm{C}$ in the presence of $5 \mathrm{mM}$ hydrogen peroxide or in the presence of $5 \mathrm{mM} o$-phenanthroline and $1.5 \mathrm{mM}$ $\mathrm{CuSO}_{4}$. 
Assay of insulin reduction. Insulin reduction was assayed by measuring the increase in absorbance at 650 $\mathrm{nm}$ as previously described [11-12].

Reactivation of reduced, denatured RNase and of scrambled RNase. Reduced, denatured RNase, and scrambled RNase were prepared as described previously [12]. Samples were assayed for RNase activity as described previously [12]. Reactivation of reduced RNase by redox buffers was initiated by diluting the reduced enzyme in $50 \mathrm{mM}$ Tris $\mathrm{pH} 8.2,1 \mathrm{mM} \mathrm{MgCl} 2,0.6 \mathrm{mM}$ dithiothreitol, $0.9 \mathrm{mM}$ oxidized glutathione at $23^{\circ} \mathrm{C}$, in the presence of thioredoxin or Trxsc. Reactivation of scrambled RNase was initiated by diluting scrambled RNase in $100 \mathrm{mM}$ Tris, $\mathrm{pH} 7.4,1 \mathrm{mM}$ EDTA, $60 \mu \mathrm{M}$ dithiothreitol at $23^{\circ} \mathrm{C}$ in the presence of thioredoxin or Trxsc, as indicated

Refolding of citrate synthase and $\alpha$-glucosidase. Denaturation, renaturation reactions, and activity determination were carried out at $23^{\circ} \mathrm{C}$ as described previously [13-14].

Thermal aggregation of citrate synthase. Citrate synthase aggregation was monitored by measuring the absorbance at $650 \mathrm{~nm}$ as described in [13-14].

Interaction of Trxse with R-CMLA. Binding assays of radiolabeled R-CMLA to Trxsc were done as described previously, using a Bio-Gel P-200 gel permeation column (R-CMLA was ${ }^{3} \mathrm{H}$-labeled by reductive methylation) [14].

Materials. Insulin (from bovine pancreas), RNaseA, thioredoxin (from Spirufina sp.), citrate synthase (from porcine heart), $\alpha$-glucosidase (from yeast), R-CMLA, bovine serum albumin, ovalbumin and lysozyme were from Sigma. All other products were reagent grade and were also obtained from Sigma. DEAE-Sephacel was from Pharmacia Fine Chemicals, and hydroxyapatite (Bio-Gel HTP) was from BioRad. The molecular weight marker kit for gel filtration chromatography was from Sigma. 


\section{RESULTS}

YbbN/Trxsc is a thioredoxin-like protein. The N-terminal sequence of $E$. coli YbbN displays a strong homology with E. coli thioredoxin 1 (TrxA) and thioredoxin 2 (TrxC) (34\% and 33\% identities, respectively) (Figure 1A): Several of the aminoacids which are conserved in different thioredoxins and are important for their structure and function [15] are conserved in YbbN. These include (Trx1 numbering) Leu-24 and Phe-27 (structural), Trp-28, Cys-35 (one of the redox cysteines), Pro-40 (maintains the conformation of the redox disulfide of Trx1), Tyr-49, Lys-57 (influences the pKa of the active Trx1 cysteines), Asn/Asp-59 and Asp-61 (clustered with the redox active site of Trx1), Ala-67, Arg-73 and Ile-75 (structural), Pro-76 (in van der Waals contacts with the Trx1 disulfide bridge), Thr-77 and Gly-84 (structural) and Gly-92 (protein interaction area).

YbbNs from E. coli and many other bacteria do not possess the canonical Cys-X-X-Cys active site which catalyzes protein disulfide interchange, but instead a Ser-X-X-Cys site. However, YbbNs from several bacteria possess the canonical $\mathrm{CxxC}$ site, suggesting that they function as protein oxidoreductases (Figure 1B). Interestingly, all members of the Ser-X-X-Cys group possess an additional cysteine (located at position of Cys-63 in E. coli YbbN), which is absent from thioredoxins and YbbNs with a CxxC site. This extra cysteine (Cys-63 in E. coli YbbN) is close to Cys-38 in the 3D model of the thioredoxin domain of YbbN / Trxsc (Figure 1C), suggesting that it may have a redox functions. A distance of $7.9 \AA$ between the sulfur atoms of Cys-38 and Cys-63 was measured on this model, which is similar to that of $6.8 \AA$ between the sulfur atoms of Cys-32 and Cys-35 in reduced Trx1 [16-17].

Purification of YbbN/Trxsc. The $y b b N$ gene of $E$. coli was amplified by polymerase chain reaction, and cloned in a pET21a vector (Novagen), under the transcriptional control of the bacteriophage T7 promoter and lac operator. A BL21(DE3) strain transformed with this vector and induced with IPTG accumulates large amounts of a soluble protein migrating in SDS-PAGE with an apparent molecular weight of around 35 $\mathrm{kDa}$, which matches the expected YbbN/Trxsc molecular weight of $32 \mathrm{kDa}$ (Figure 2, lane 2). The overexpressed protein is not detectable in an uninduced extract (Figure 2, lane 1), and represents $40 \%$ in mass of the proteins of the induced extract. The overexpressed protein is not detectable in membrane fractions (pellet after a 200,000 x g centrifugation for 1 hour (not shown)). Ybbn / Trxsc was purified to homogeneity by two chromatographic steps on a DEAE-Sephacel ion exchange column (Figure 2, lane 3) and on a hydroxyapatite column (Figure 2, lane 4), as described under "Materials and Methods" Its identity was confirmed by mass spectrometry analysis (not shown), and it was designated Trxsc (for thioredoxin with a Ser-X-X-Cys site).

Reduced and oxidized Trxsc. Protein disulfide isomerases such as thioredoxin, PDI and DsbA contain a CxxC sequence in their active site and shift, upon oxidation, from a dithiol state to a state containing an intramolecular disulfide [18]. Trxsc contains two cysteines, Cys-38 and Cys-63, which are close together in 
the 3D model. We titrated the thiol groups of reduced and oxidized Trxsc with DTNB (in the absence of guanidine hydrochloride). Reduced Trxsc contains 1.9 SH titratable groups per mol (not shown); this reflects the presence of the two cysteines, and suggests that they are both exposed to the solvent in the native state. Oxidized Trxcs (obtained by incubation of reduced Trxsc for 1 hour in the presence of $5 \mathrm{mM}$ hydrogen peroxide, or in the presence of $5 \mathrm{mM}$ o-phenanthroline and $1.5 \mathrm{mM} \mathrm{CuSO}_{4}$ [19] contains $0.3 \mathrm{SH}$ groups per mol (not shown), suggesting that, upon oxidation, 1.6 thiol groups of Trxsc are oxidized. These results are similar to those obtained with $E$. coli $\operatorname{Tr} x 1$ (1.8 SH in reduced thioredoxin and 0.2 in oxidized thioredoxin [20], and suggest that Trxsc undergoes a dithiol-disulfide transition upon oxidation. In SDS polyacrylamide gels, both the reduced and oxidized forms of Trxsc migrate as monomers (Figure 2B). These results suggest that Cys-38 and Cys-63 in native Trxsc are exposed to the solvent and form an intramolecular disulfide bridge upon oxidation. Formation of a disulfide bridge between Cys-38 and Cys-63 is consistent with the 7.9 $\AA$ span between their sulfur atoms (Figure 1C), similar that (6.8 $\AA$ ) between the vicinal Cys-32 and Cys-35 in reduced Trx1 [16-17].

Oligomeric forms of reduced and oxidized Trxsc. Reduced and oxidized Trxsc were analyzed by size exclusion chromatograhy on a SW G-3000 HPLC column, as described under "Materials and Methods". Reduced Trxsc migrates as a dimer with an apparent molecular weight of around 65,000 Da, whereas oxidized Trxsc migrates as a mixture of tetramers, hexamers and higher molecular weight oligomers (Figure 2C). Thus, Trxsc oxidation results in changes in its oligomeric structure with the formation of higher molecular weight oligomers. The existence of dynamic oligomers has been reported for several chaperones, including DnaK [21] and small Hsps.

Oxidative folding of reduced, denatured Rnase, and refolding of scrambled RNase. Protein disulfide isomerases catalyze three typical redox reactions in vitro: Oxidative folding of reduced RNase [11], refolding of scrambled RNase [18,22] and reduction of insulin disulfide bridges [23]. Trxsc stimulates several-fold the reactivation of reduced RNaseA (21\% reactivation after 1 hour, compared to $8 \%$ in the absence of added catalyst and 48\% in the presence of thioredoxin) (Figure 3A); the chaperone DnaK was unable to catalyze this reactivation (not shown), suggesting that a chaperone function is not sufficient to catalyze the reactivation of reduced, denatured RNase.

Scrambled RNaseA is fully oxidized but inactive as a catalyst of RNA hydrolysis owing to the formation of a complex mixture of species containing an undefined variety of (mainly) intramolecular disulphide bonds. It is obtained by oxidation of reduced RNaseA in the presence of guanidine hydrochloride, and necessitates an interchange of disulfide bonds to acquire the native conformation. Thioredoxin [11], PDI [18] or DsbC [23] can reactivate scrambled RNase in the presence of dithiothreitol. Trxsc reactivates scrambled RNase (three times less efficiently than thioredoxin) whereas dithiothreitol alone is unable to reactivate scrambled RNase significantly (Figure 3B). Thus, also Trxsc displays a protein disulfide isomerase activity. 
Reduction of insulin disulfide bonds. Thioredoxin [11], PDI [20] and DsbA [24] catalyze the dithiothreitol-dependent reduction of insulin. Trxsc was unable to stimulate insulin precipitation in a dithiothreitol-dependent manner, whereas thioredoxin led to insulin precipitation after only 20 min (Figure 3C). Trxsc shares this inability to reduce insulin disulfide bonds with glutaredoxins 2 and 3 [17, 25].

Trxsc chaperone properties: Renaturation of unfolded proteins. Since many protein oxidoreductases display chaperone properties [26-29], we investigated the chaperone properties of Trxsc. Chaperones are implicated in protein folding, protein targeting to membranes, protein renaturation or degradation after stress, and in the control of protein-protein interactions (reviewed in [30, 28]). Citrate synthase and $\alpha$-glucosidase, whose refolding is facilitated by GroEL, DnaK, Hsp90 and small Hsps [31, 13-14], were chosen as substrates for this reaction. The refolding yield of citrate synthase (after denaturation in $8 \mathrm{M}$ urea) is increased from $7 \%$ in the absence of added proteins to $23 \%$ in the presence of Trxsc, $28 \%$ in the presence of DnaK (Figure $4 \mathbf{A}$ ), and $36 \%$ in the presence of the DnaK/DnaJ/GrpE/ATP chaperone machine (not shown). Half-maximal reactivation occurs at $0.3 \mu \mathrm{M}$ Trxsc, which is similar to or lower than the concentration of DnaK [14], thioredoxin (Figure 4B, [26]) and Hsp25 [13], required for half-maximal reactivation of citrate synthase under similar conditions. Control proteins such as ovalbumin and lysozyme were unable to stimulate citrate synthase renaturation, while serum albumin could stimulate it to some extent (Figure 4B). In similar experiments, the refolding yield of $0.3 \mu \mathrm{M}_{\alpha}$-glucosidase was increased from $12 \%$ in the absence of Trxsc to $86 \%$ in the presence of $2 \mu \mathrm{M}$ Trxsc (Figure 4C) (and 36\% in the presence of $2 \mu \mathrm{M}$ DnaK (not shown)).

In contrast with DnaK [32] and small Hsps [13], Trxsc $(2-30 \mu \mathrm{M})$ does not protect $0.8 \mu \mathrm{M}$ citrate against thermal aggregation at $43^{\circ} \mathrm{C}$, although Trxsc is stable at this temperature (not shown). This suggests that, like thioredoxin which does not protect citrate synthase against thermal aggregation either [29], Trxsc interacts more efficiently with unfolded proteins such as urea-denatured citrate synthase than with partially unfolded proteins such as citrate synthase at $43^{\circ} \mathrm{C}$.

YbbN/ Trxsc, like other chaperones, interacts preferentially with unfolded proteins. Complex formation between R-CMLA (a permanently unfolded protein [32]) and Trxsc was analyzed by gel filtration on a Biogel P-200 column equilibrated with $50 \mathrm{mM}$ Tris- $\mathrm{HCl} \mathrm{pH}$ 8.2, $50 \mathrm{mM} \mathrm{KCL}, 1 \mathrm{mM}$ dithiothreitol, $100 \mu \mathrm{g} / \mathrm{ml}$ bovine serum albumin. When R-CMLA is filtered in the presence of Trxsc, $28 \%$ of R-CMLA fractionates as a high molecular weight complex (Figure 4D). Similarly, DnaK binds 31\% of added R-CMLA (Figure 4D). Thus, Trxsc interacts strongly with R-CMLA, in contrast with thioredoxin which does not form stable complexes with unfolded proteins [29], suggesting that Trxsc interacts with unfolded proteins more efficiently than thioredoxin. 


\section{DISCUSSION}

We have cloned, purified, and characterized YbbN/Trxsc as a weak protein disulfide oxidoreductase and a molecular chaperone. Trxsc migrates as a single band at $35 \mathrm{kDa}$ in an SDS-polyacrylamide gel, and the molecular weight of the native reduced protein, estimated by gel filtration and by ultracentrifugation, is close to $65,000 \mathrm{kDa}$, suggesting that it is a homodimer.

In E. coli Trxsc, the canonical CxxC active site of thioredoxins is replaced by a SxxC sequence. Our results suggest, however, that Trxsc functions with two redox active cysteines, Cys-38 and Cys-63: the two cysteines of native, reduced YbbN are titrated by DTNB and oxidized by hydrogen peroxide without the formation of intermolecular disulfides, suggesting that they form an internal disulfide. Chloroplast thioredoxin $\mathrm{f}$ displays a cysteine residue (Cys-73) which is located at the same position as Trxsc Cys-63 in the thioredoxin structure, but possesses a CGPC active site like bona fide thioredoxins. Cys-73 of thioredoxin $\mathrm{f}$ is surface exposed and close to the active site disulfide [33], but it has no redox function (the redox function is accomplished by the two cysteines of the CGPC active site); it is important for the interaction between thioredoxin $\mathrm{f}$ and its substrate proteins and it is involved in the formation of thioredoxin f dimers upon oxidation [34]. Human thioredoxin also possesses an extra cysteine (Cys-73) in addition to its CGPC cysteines, but its localization in the structure is entirely different from that of $\mathrm{YbbN}$ [35], and its role has not been clarified.

YbbN displays several protein oxidoreductase activities, significantly lower than those of thioredoxin [11], PDI [18], DsbA [24] or DsbC [23] It catalyzes the oxidation of reduced, denatured RNase by redox buffers and the isomerisation of scrambled RNase, with a 3-5 fold lower activity than thioredoxin. The lower efficiency of Trxsc might be explained by a lower redox efficiency of its cysteines, and might reflect a possibility for a modulation of Trxsc activity by conformational changes induced by substrates, cytoplasmic co-factors or physiological conditions. In contrast with many thioredoxins, Trxsc is unable to stimulate insulin reduction in the presence of dithiothreitol. The unability of Trxsc to reduce insulin disulfide bonds (like glutaredoxins 2 and $3[17,25]$ ) probably reflects an unfavorable enzyme substrate interaction and/or an elevated redox potential of $\mathrm{YbbN}$.

Trxsc behaves as a molecular chaperone. It increases 3-fold the yield of active citrate synthase renaturation (like other molecular chaperones), and 7-fold the yield of $\alpha$-glucosidase renaturation. The stimulation factor of protein renaturation, and the Trxsc concentrations required for half-maximal renaturation (below $1 \mu \mathrm{M}$ ), are not significantly different from those of [14], Hsp90 [13], thioredoxin [29] and small Hsps [31]. In contrast with several chaperones, but like thioredoxin, Trxsc does not protect citrate synthase from thermal denaturation, suggesting that it interacts more efficiently with unfolded proteins than with partially denatured proteins. Trxsc forms stable complexes with the unfolded protein R-CMLA, suggesting that it possesses a higher affinity for unfolded proteins than thioredoxin, which is unable to form such complexes [29]. Thus, YbbN displays chaperone properties and weak redox properties in vitro. In vivo experiments will allow to determine the importance of each of these properties in the cellular physiology. 
ACKNOWLEDGEMENTS. The authors thank Mederic Diard for his help during the early course of this work, and A. Kropfinger for correction of the English language. This work was supported by grant CR 521090 from the DGA to G.R.

\section{REFERENCES}

[1] E.S. Arner, A. Holmgren, Physiological functions of thioredoxin and thioredoxin reductase, Eur. J. Biochem. 26 (2000) 6102-6109.

[2] A. Miranda-Vizuete, A.E. Damdidopoulos, J. Gustafsson, G. Spyrou, Cloning, overexpression, and characterization of a novel Escherichia coli thioredoxin, J. Biol. Chem. 272 (1997) 30841-30837.

[3] D. Ritz, H. Patel, B. Doan, M. Zheng, F. Aslund, G. Storz, J. Beckwith, Thioredoxin 2 is involved in the oxidative stress response in Escherichia coli, J. Biol. Chem. 275 (2000) 2505-2512.

[4]A. Holmgren, Thioredoxin and glutaredoxin systems. J. Biol. Chem. 264 (1989)13963-13966.

[5] H.F. Gilbert, Molecular and cellular aspects of thiol-disulfide exchange, Adv. Enzymol. Relat. Areas Mol. Biol. 63(1990) 69-172.

[6] A.I. Derman, W.A. Prinz, D. Belin, J. Beckwith, Mutations that allow disulfide bond formation in the cytoplasm of Escherichia coli, Science 262 (1993) 1744-1747

[7] L. Debarbieux, J. Beckwith, Electron avenue: pathways of disulfide bond formation and isomerization, Cell. 99 (1999) 117-119.

[8] W.A. Prinz, F. Aslund, A. Holmgren, J. Beckwith, The role of the thioredoxin and glutaredoxin pathways in reducing protein disulfide bonds in the Escherichia coli cytoplasm, J. Biol. Chem. 272 (1997) 15661-15667.

[9] J.F. Collet, J.C. D'Souza, U. Jakob, J.C. Bardwell, Thioredoxin 2, an oxidative stress-induced protein, contains a high affinity zinc binding site, J. Biol. Chem. 278 (2003): 45325-45332.

[10] D.E. Fomenko, V.N. Gladyshev, Identity and functions of CxxC-derived motifs, Biochemistry 42 (2003) 11214-11225.

[11] V.P. Pigiet, B.J. Schuster, Thioredoxin-catalyzed refolding of disulfide-containing proteins, Proc. Natl. Acad. Sci. U.S.A. 83(1986) 7643-7647.

[12] A. de Crouy-Chanel, M. Kohiyama, G. Richarme, A novel function of Escherichia coli chaperone DnaJ, J. Biol. Chem. 270 (1995) 22669-22672.

[13] U. Jakob, M. Gaestel, K. Engel, J. Buchner, Small heat shock proteins are molecular chaperones. J. Biol. Chem. 268 (1993) 1517-1520.

[14] G. Richarme, T.D. Caldas, Chaperone properties of the bacterial periplasmic substrate-binding proteins, J. Biol. Chem. 272 (1997) 15607-15612.

[15] H. Eklund, F.K. Gleason, A. Holmgren, Structural and functional relations among thioredoxins of different species, Proteins 11 (1991) 13-28. 
[16] H.J. Dyson, G.P. Gippert, D.A. Case, A. Holmgren, .P.E.Wright, Three-dimensional structure of the reduced form of Escherichia coli thioredoxin determined by nuclear magnetic resonance spectroscopy, Biochemistry 29 (1990) 4129-4136.

[17] J.D. Forman-Kay, G.M. Clore, P.T. Wingfield, A.M. Gronenborn, $1 \mathrm{H}$ and $15 \mathrm{~N}$ resonance assignments and secondary structure of the human thioredoxin C62A, C69A, C73A mutant, Biochemistry 30 (1991) 26852698.

[18] H.C. Hawkins, E.C. Balckburn, R.B. Freedman, Comparison of the activities of protein disulphide isomerase and thioredoxin in catalysing disulphide isomerization in a protein substrate, Biochem. J. 275 (1991) 349-353.

[19] C.L. Careaga, J.J. Falke, Thermal motions of surface alpha-helices in the D galactose chemosensory receptor. Detection by disulfide trapping, J. Mol. Biol. 226 (1992) 1219-1235.

[20] P.T. Chivers, R.T. Raines, General acid/base catalysis in the active site of Escherichia coli thioredoxin, Biochemistry 36 (1997) 15810-15815.

[21] H.J. Schönfeld, D. Schmidt, H. Schroder, B. Bukau, The DnaK chaperone system of Escherichia coli: quartenary structures and interactions of the DnaK and GrpE components, J. Biol. Chem. 270 (1995) 21832189.

[22] M. Russel, P. Model, The role of thioredoxin in filamentous phage assembly. Construction, isolation and characterization of mutant thioredoxins, J. Biol. Chem. 261(1986) 14997-15004.

[23] Y. Akiyama, S. Kamitani, N. Kusukawa, K. Ito, In vitro catalysis of oxidative folding of disulfide-bonded proteins by the Escherichia coli dsbA gene product, J. Biol. Chem. 267 (1992) 22440-22445.

[24] J.C.A. Bardwell, K. McGovern, J. Beckwith, Identification of a protein required for disulfide bond formation in vivo, Cell 67 (1991) 581-589.

[25] A. Vlamis-Gardikas, F. Aslund, G. Spyrou, T. Bergman, A. Holmgren, Cloning, overexpression, and characterization of glutaredoxin 2, an atypical thioredoxin from Escherichia coli, J. Biol. Chem. 272 (1997) 11236-11243.

[26] F. Shao, M.W. Bader, U. Jakob, J.C. Bardwell, DsbG, a protein disulfide isomerase with chaperone activity, J. Biol. Chem. 275 (2000) 13349-13352.

[27] B. Tsai, C. Rodighiero, W.I. Lencer, T.A. Rapoport, Protein disulfide isomerase acts as a redox-dependent chaperone to unfold cholera toxin, Cell 104 (2001) 937-948.

[28] F.U. Hartl, M. Hayer-Hartl, Molecular chaperones in the cytosol: from nascent chains to folded proteins, Science 195 (2002) 1852-1858.

[29] R. Kern, A. Malki, A. Holmgren, G. Richarme, Chaperone properties of Escherichia coli thioredoxin and thioredoxin reductase, Biochem. J. 371(2003) 965-972.

[30] D.A. Dougan, A. Mogk, B. Bukau, Protein folding and degradation in bacteria: to degrade or not to degrade? That is the question, Cell Mol. Life Sci. 59 (2002) 1607-1616.

[31] J. Buchner, M. Schmidt, M. Fuchs, R. Jaenicke, R. Rudolph, F.X. Schmid, T. Kiefhaber, GroE facilitates refolding of citrate synthase by suppressing its aggregation, Biochemistry 30 (1991) 1586-1591. 
[32] K. Liberek, D. Skowyra, M. Zylicz, C. Johnson, C. Georgopoulos, The DnaK chaperone changes conformation upon ATP hydrolysis, thus triggering its dissociation from a bound target protein, J. Biol. Chem. 266 (1991) 14491-14496.

[33] G. Capitani, Z. Markovic-Housley, G. DelVal, M. Morris, J.N. Jansonius, P. Schürmann, Crystal structure of two functionally different thioredoxins in spinach chloroplasts, J. Mol. Biol. 302 (2000) 135-154.

[34] M.K. Geck, F.W. Larimer, F.C. Hartman, The molecular pathway for the regulation of phosphoribulokinase by thioredoxin f, J. Biol. Chem. 271(1996) 24736-24740.

[35] J.F. Andersen, D.A.R. Sanders, J.R. Gasdaska, A. Weichsel, G. Powis, W.R. Montfort, Human thioredoxin homodimers : regulation by $\mathrm{pH}$, role of aspartate 60, and crystal structure of the aspartate 60arginine mutant, Biochemistry 36 (1997) 13979-13988.

[36] N. Guex, M.C. Peitsch, SWISS MODEL and the Swiss Pdb viewer : an environment for comparative protein modeling, Electrophoresis 18 (1997) 2714-2723. 


\section{LEGENDS TO FIGURES}

Figure 1. YbbN / Trxsc is a thioredoxin-like protein. A) Multiple sequence alignment of $E$. coli thioredoxin 1 (TrxA), thioredoxin $2(\operatorname{TrxC})$ and of the N-terminal sequence of YbbN / Trxsc. Conserved aminoacids in the three sequences are indicated by a star, conservative homologies by two dots and semiconservative homologies by a single dot. The redox active cysteines of $\operatorname{Trx} 1$ and $\operatorname{Trx} 2$ are boxed, as well as the Cys-38 and Cys-63 of Trxsc. The alignment was done with CLUSTALW (Thomson et al., 1994). B) Multiple sequence alignments of the N-terminal domains of YbbNs from different bacteria. The cysteines of the $\mathrm{SxxC}$ or $\mathrm{CxxC}$ site, and the extra cysteine corresponding to Cys-63 of E. coli YbbN are colored in black. The alignment was done with HOBACGEN (Perriere et al., 2000). C) Cartoon representation of the spatial structure of the N-terminal domain of YbbN / Trxsc. The positions of the sulfur atoms of Cys-38 and Cys-63 are labeled. We used the SWISS-MODEL for the 3D modelization (http ://www.expasy.org/spdbv/) [36].

Figure 2. A) Purification of $\mathrm{YbbN} /$ Trxsc. Protein samples were separated by sodium dodecylsulfate polyacrylamide gels (12\%), and stained with Coomassie brillant blue. Lane 1, crude extract from uninduced BL21(DE3), pET-21aYBBN; lane 2, crude extract from BL21(DE3), pET-21aYBBN induced by IPTG; lane3, YbbN/Trxsc pool from the DEAE-Sephacel column; lane4, YbbN/Trxsc pool from the hydroxyapatite column. The positions of molecular weight markers are indicated on the left. B) Migration of reduced and oxidized Trxsc in an SDS polyacrylamide gel under non reducing conditions. Reduced Trxsc (lane 1) and oxidized Trxsc ( lane 2) were dissolved in SDS sample buffer (50 mM Tris $\mathrm{pH} 6.8,1.25 \%$ SDS, Bromophenol Blue, without dithiothreitol, and run on a $10 \%$ polyacrylamide gel. The gel was stained with Coomassie Blue. The positions of molecular weight markers are indicated on the left. C) Oligomeric forms of reduced and oxidized Trxsc. Reduced (thick line) or oxidized (thin line) Trxsc ( $2 \mathrm{mg} / \mathrm{ml}$ ) were loaded on a TSK-G3000-SW HPLC gel permeation column as described under "Materials and Methods", and eluted at a rate of $0.5 \mathrm{ml} / \mathrm{min}$. The peak represents the absorbance of Trxsc at $280 \mathrm{~nm}$, and the marks indicate the elution of $\alpha_{\alpha}$-amylase (molecular weight, 200,000 Da) alcohol dehydrogenase (molecular weight, 150,000 Da) bovine serum albumin (molecular weight, 66,000 Da) and carbonic anhydrase (molecular weight, 29,000 Da) run in a separate experiment.

Figure 3. Protein disulfide oxidoreductase activities of Trxsc. A) Oxidative folding of reduced, denatured RNase in the presence of redox buffers. The mixtures $(0.6 \mathrm{mM}$ dithiotreitol, $1 \mathrm{mM}$ oxidized glutathione, 1 $\mathrm{mM} \mathrm{MgCl} 2,50 \mathrm{mM}$ Tris $\mathrm{pH} 8.2$ at $23^{\circ} \mathrm{C}$ ) containing $30 \mu \mathrm{M}$ reduced, denatured RNase alone or in the presence of $50 \mu \mathrm{M}$ Trxsc or $50 \mu \mathrm{M}$ thioredoxin were assayed for RNase activity after 1 hour renaturation. B) Refolding of scrambled RNase. The mixtures $\left(100 \mathrm{mM}\right.$ Tris $\mathrm{pH} 7.4,1 \mathrm{mM}$ EDTA at $\left.23^{\circ} \mathrm{C}\right)$ containing 60 $\mu \mathrm{M}$ dithiothreitol, and $30 \mu \mathrm{M}$ scrambled RNase alone (open circles) or in the presence of $50 \mu \mathrm{M}$ Trxsc (closed circles) or $50 \mu \mathrm{M}$ thioredoxin (triangles) were assayed for RNase activity at the times indicated. C) 
Insulin reduction by dithiothreitol. The incubation mixtures contained $100 \mathrm{mM} \mathrm{N}_{2}$-equilibrated potassium phosphate pH 6.6. 0.3 mM EDTA. $0.13 \mathrm{mM}$ bovine insulin and $0.3 \mathrm{mM}$ dithiothreitol alone (open circles). or in the presence of $10 \mu \mathrm{M}$ Trxsc (closed circles) or $1 \mu \mathrm{M}$ thioredoxin (triangles). The reduction of insulin and its resulting precipitation were monitored by following optical density at $650 \mathrm{~nm}$.

Figure 4. Chaperone properties of Trxsc A) Kinetics of citrate synthase refolding: citrate synthase was denatured in urea and then renatured by dilution of the denaturant as described under «Materials and Methods», at a concentration of $0.1 \mu \mathrm{M}$ in the absence of additional protein (open circles) and in the presence of either $3 \mu \mathrm{M}$ Trxsc (filled circles) or $3 \mu \mathrm{M}$ DnaK (filled triangles). B) Dependence of citrate synthase refolding on Trxsc concentration: citrate synthase was denatured in urea and subsequently renatured for $20 \mathrm{~min}$ by dilution of the denaturant in the presence of Trxsc (filled circles) or thioredoxin (filled triangles) at the indicated concentrations. As a control, we measured citrate synthase renaturation in the presence of lysozyme (open circles), ovalbumin (open squares) or serum albumin (crosses). C) Kinetics of $\alpha$ glucosidase refolding: $\alpha$-glucosidase was denatured in urea and then renatured by dilution of the denaturant as described under «Materials and Methods», at a concentration of $0.1 \mu \mathrm{M}$ in the absence of additional protein (open circles) and in the presence of $2 \mu \mathrm{M}$ Trxsc (filled circles). D) Gel permeation chromatography of Trxsc / R-CMLA complexes. $2 \mu \mathrm{M}$ of ${ }^{3} \mathrm{H}$-R-CMLA was incubated either alone (crosses), or with $6 \mu \mathrm{M}$ reduced Trxsc (filled circles), $6 \mu \mathrm{M}$ oxidized Trxsc (open circles) or $6 \mu \mathrm{M}$ DnaK (filled squares), and the mixture was loaded on a Biogel P200 column as described under « Materials and Methods ». Fractions were collected and counted for radioactivity. The amount of bound ligand was estimated on the basis of the amount of ligand in the fractions that did not overlap with the distribution of free ligand. 


\begin{tabular}{|c|c|c|}
\hline & & $\begin{array}{rrr}10 & 20 \\
1 & 1\end{array}$ \\
\hline $\mathrm{p} 00274$ & $\operatorname{TrxA}$ & - - - n \\
\hline $\mathrm{p} 33636$ & $\operatorname{TrxC}$ & MNTVCTHCQA INRI PDDRIEDAAKCGRCGHDLFDGEVINATGETLD - KLLKDDLPVVIDF \\
\hline p 77395 & Ybbl & 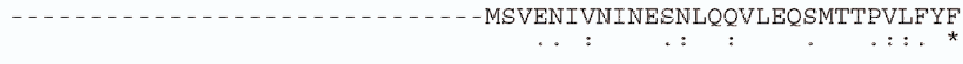 \\
\hline & & $\begin{array}{ccccc}40 & 50 & 60 & 70 & 80 \\
1 & 1 & 1 & 1\end{array}$ \\
\hline $\mathrm{p} 00274$ & $\operatorname{TrxA}$ & WAEWFGFCKMIAPI LDEI ADEYQGKLTVAKLNIDQNPGTAPKYGIRG I PTLLLFKNGEVA \\
\hline p33636 & $\operatorname{TrxC}$ & WAPWGGFGRNFAPIFEDVAQERSGKVRFVKVNTEAERELSSRFGIRS I PTIMI FKNGQVV \\
\hline p77395 & YbaN & 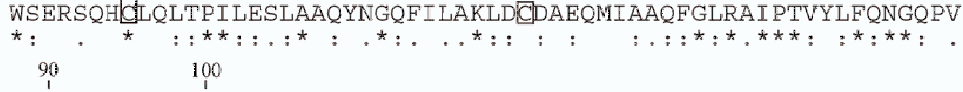 \\
\hline $\mathrm{p} 00274$ & TrxA & ATKVGALSKGQLKEFLDANLA \\
\hline p33636 & $\operatorname{TrxC}$ & DNLNGAVPKAPFDSWLNESL- \\
\hline p77395 & YbbN & $\begin{array}{c}\text { DGFQGPQPEEAIRALLDKVLP } \\
\star *: \quad: \quad *: * *\end{array}$ \\
\hline
\end{tabular}

\section{Figure $1 \mathrm{~A}$}

E.coli

S.typhimurium

S.enterica

$Y$.pestis

P. Iuminescens

A.tumefaciens

R.melizoti

R. Ioti

C.crescentus

B. solanacearum

S. aureofaciens

P. aeruginosa

X.fastidiosa

AAC73594
AAL19458
CAD04989
CAC92324
Q9AHZ9
AAK89705
Q92L96
Q98E31
Q9ABWO
CAD14481
Thio_strau
Q9HWW7
Q9PBHO

Figure 1B

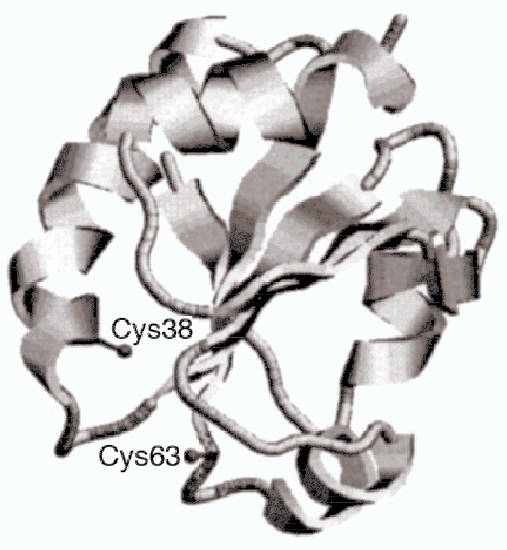

Figure 1C 


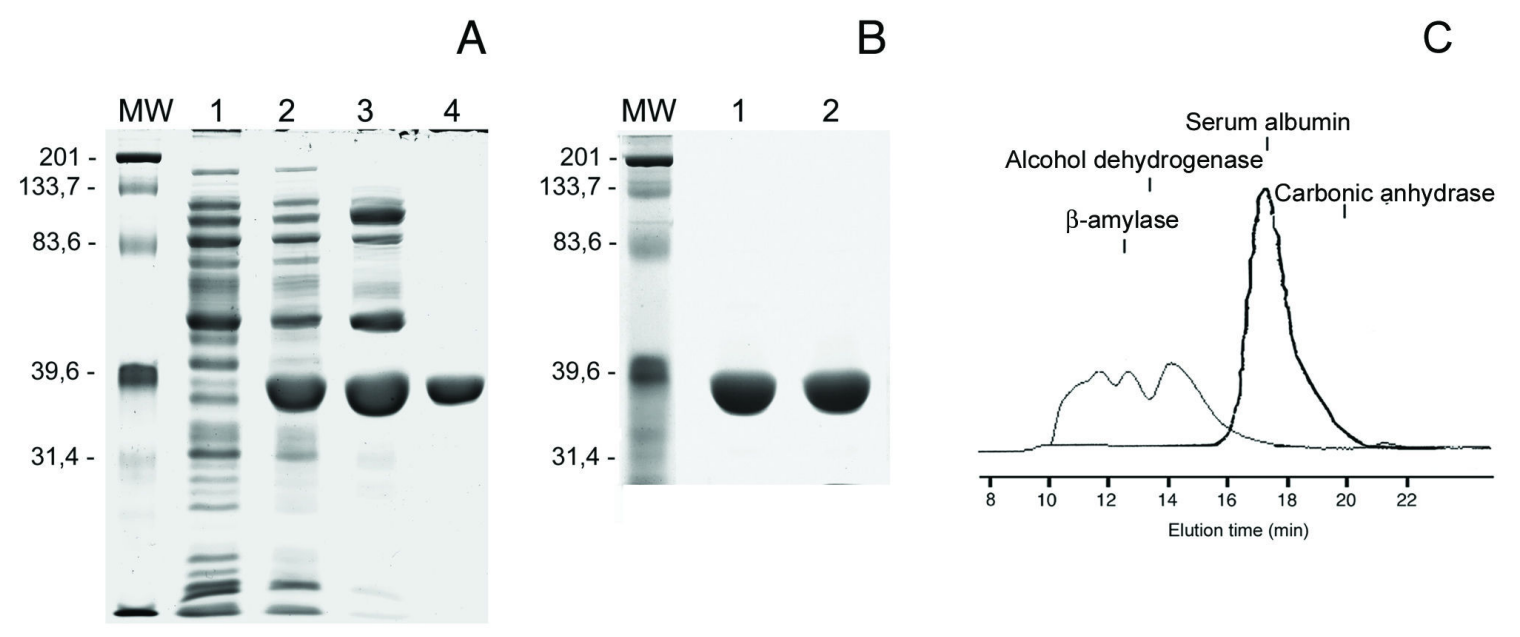

Figure 2 

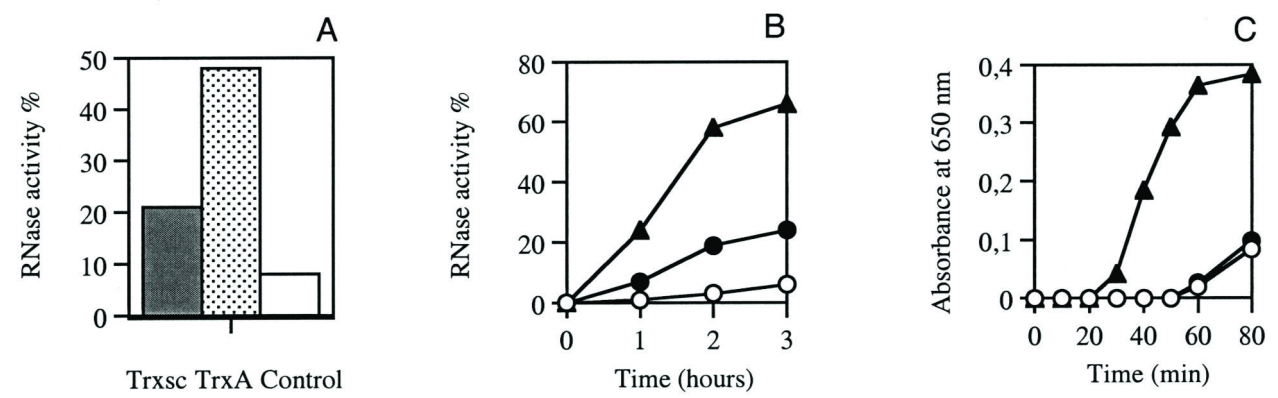

Figure 3 

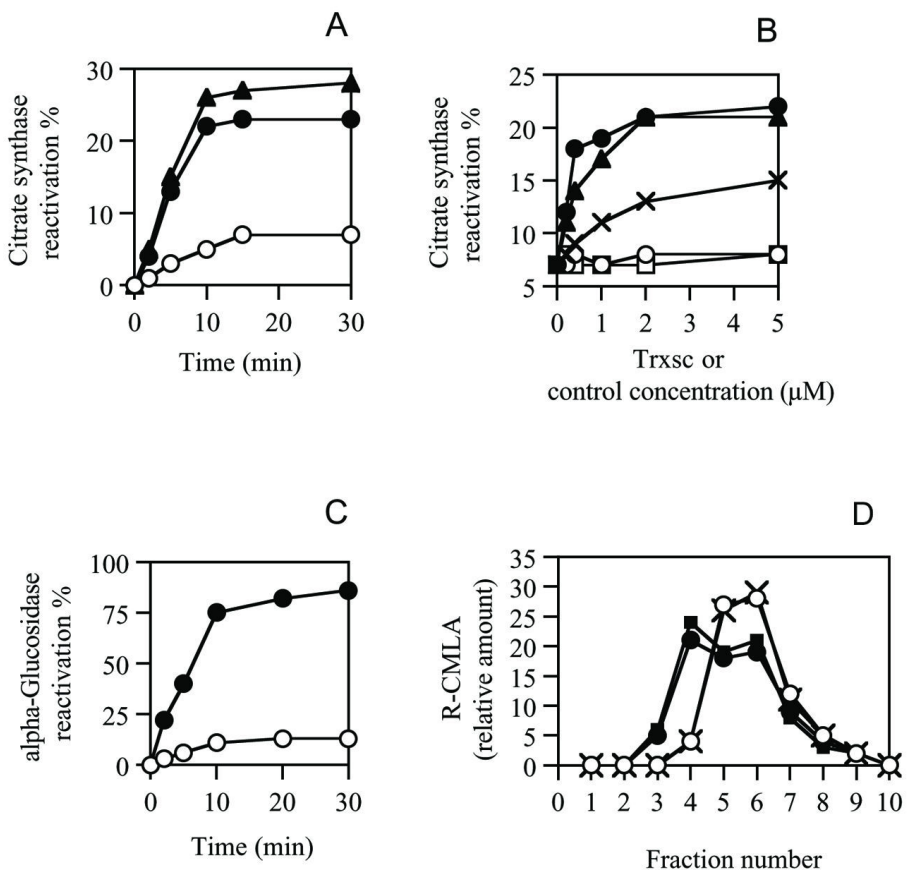

Figure 4 